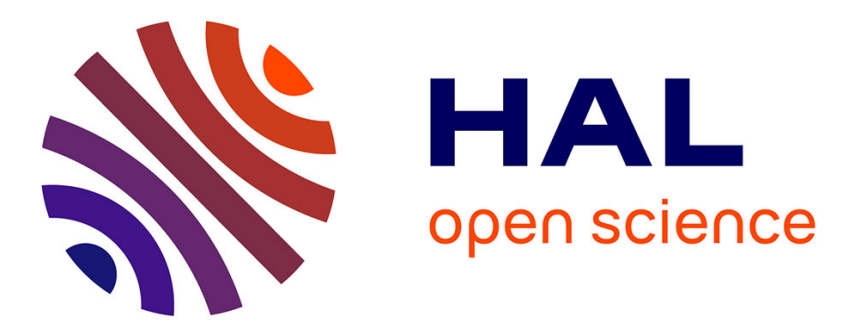

\title{
Modelling of a hysteresis motor using the Jiles-Atherton model
}

\author{
Abdelkader Benabou, Lounas Bouaziz, Stéphane Clenet
}

\section{To cite this version:}

Abdelkader Benabou, Lounas Bouaziz, Stéphane Clenet. Modelling of a hysteresis motor using the Jiles-Atherton model. European Physical Journal: Applied Physics, 2004, 29 (3), pp.259-265. 10.1051/epjap:2004224 . hal-01203640

\section{HAL Id: hal-01203640 \\ https://hal.science/hal-01203640}

Submitted on 21 Jun 2017

HAL is a multi-disciplinary open access archive for the deposit and dissemination of scientific research documents, whether they are published or not. The documents may come from teaching and research institutions in France or abroad, or from public or private research centers.
L'archive ouverte pluridisciplinaire HAL, est destinée au dépôt et à la diffusion de documents scientifiques de niveau recherche, publiés ou non, émanant des établissements d'enseignement et de recherche français ou étrangers, des laboratoires publics ou privés. 


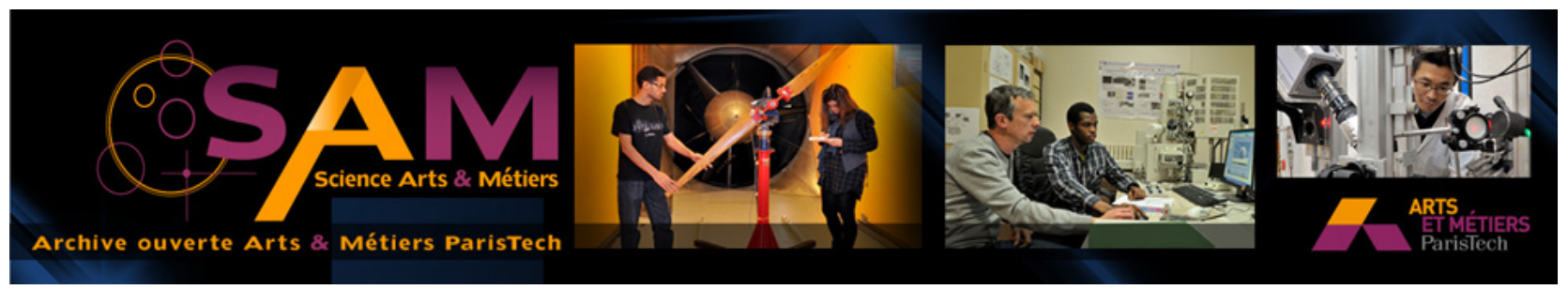

Science Arts \& Métiers (SAM)

is an open access repository that collects the work of Arts et Métiers ParisTech researchers and makes it freely available over the web where possible.

This is an author-deposited version published in: http://sam.ensam.eu

Handle ID: .http://hdl.handle.net/10985/10139

\section{To cite this version :}

Abdelkader BENABOU, Stéphane CLENET, Lounas BOUAZIZ - Modelling of a hysteresis motor using the Jiles-Atherton model - European Physical Journal - Applied Physics - Vol. 29, $n^{\circ} 3$, p.259-265 - 2015 
EPJ manuscript No.

(will be inserted by the editor)

\title{
Modelling of a hysteresis motor using the Jiles-Atherton model
}

\author{
Abdelkader Benabou ${ }^{1}$, Lounas Bouaziz ${ }^{2}$ and Stéphane Clénet $^{3 a}$ \\ 1 L2EP, Bâtiment P2, USTL, 59655 Villeneuve d'Ascq, France \\ 2 Société PRECILEC, 41-47 rue Guynemer BP 239, 89002 Auxerre Cedex, France \\ 3 L2EP, ENSAM, 8 Boulevard Louis XIV, 59046 Lille, France
}

Received: date / Revised version: date

\begin{abstract}
In this paper, we present a model of a hysteresis motor based on Maxwell's equations coupled with the Jiles-Atherton (J-A) hysteresis model solved by the finite element method. The aim of this work is to validate such a model by comparison with the experimental results (electromagnetic torque, voltage, current). We also present an analysis of this motor when imposing current or voltage in the $2 \mathrm{D}$ vector potential formulation.
\end{abstract}

PACS. 75.60.-d Hysteresis motor, Jiles-Atherton model

\section{Introduction}

The modelling of the hysteresis phenomenon in electromagnetic devices is widely studied in electrical engineering, especially to take the iron losses into account. Several models have been proposed in the literature, for instance Preisach based models $[1,2]$. Another model based on a physical approach of the hysteresis phenomenon, the Jiles-Atherton model [3], is also often used. Previous works have shown that both kinds of model can be implemented in finite element analysis [4]. They can be used to pre-

Send offprint requests to:

a Present address: stephane.clenet@lille.ensam.fr dict accurately the iron losses and could be very useful to design electrical machines. These models are also interesting to model phenomena either like the inrush current in a transformer, due to existing remanent field in the iron core, or like electromechanical conversion based on ferromagnetic hysteresis. The hysteresis motor presents some interesting operating characteristics such as constant speed at steady state and high starting torque. Then, the hysteresis motor, connected to a fixed frequency supply network, is well adapted for constant speed applications requiring many startups. The design of this type of machine is quite difficult because of the complexity of the 
hysteresis phenomenon. Many models based on magnetic equivalent networks have been proposed in the seventies $[5,6]$, but they are not necessarily well adapted for the design. Recently, Finite Element Models taking into account hysteresis have been used to study hysteresis motors. Some are based on a post-processing calculation using a scalar hysteresis model [7] and others the vectorized Preisach model [8]. In this paper, we present a hysteresis motor model using a finite element analysis coupled with external circuit equations and using a simple hysteresis model requiring few informations about the material.

In the first section, the studied machine is presented. Then, the numerical model, and in particular the coupling with external circuit, are detailed. We use the J-A model for the hysteretic material. This model is simple to be implemented in a finite element code and is less timeconsuming than other models like the Preisach model [9]. It also requires few data for the parameter identification. In a second step, the model is validated by a comparison with measurements (torque and current). Finally, the mathematical model is used to predict and discuss the behavior of the hysteresis motor.

\section{Presentation of the system}

In this section we present the studied system from the geometrical, material and electrical points of view. The studied hysteresis motor is composed of two main parts (Figure 1): the stator and the rotor. Its rotating speed is $22500 \mathrm{rpm}$. The 3 -phase stator winding has 8 poles distributed in a total of 24 slots, which are totally closed. It

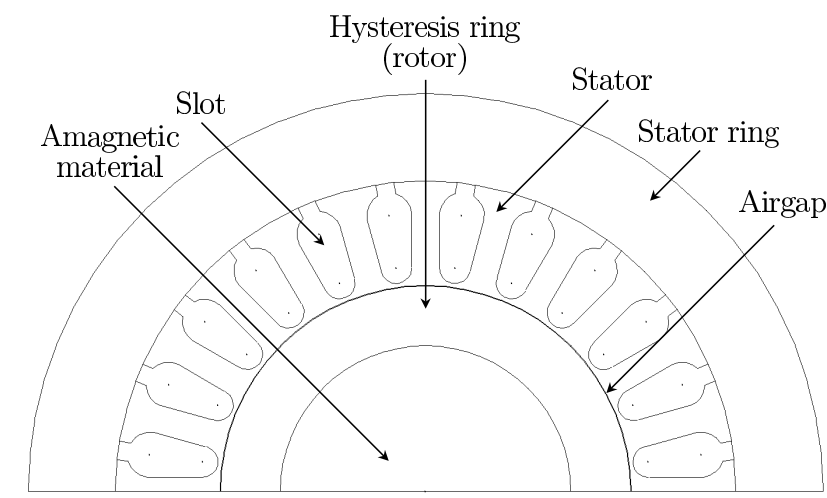

Fig. 1. Hysteresis motor geometrical structure

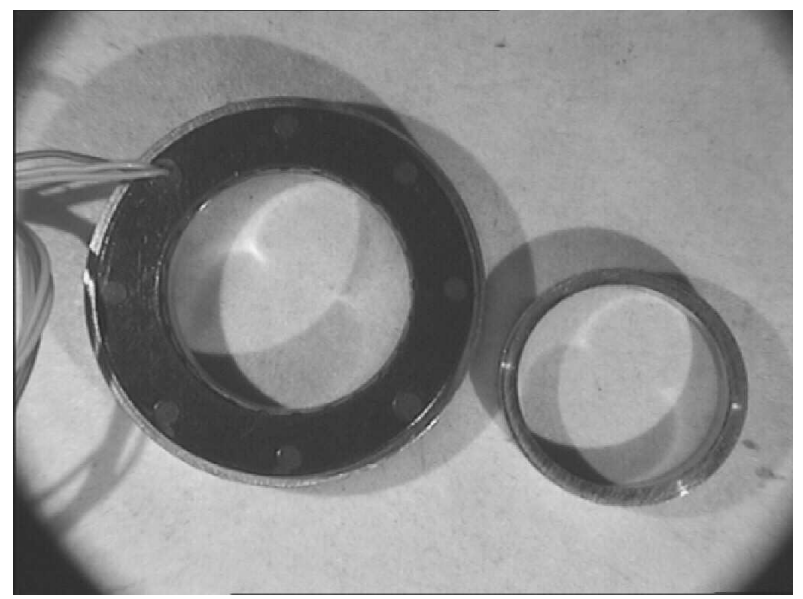

Fig. 2. Studied motor

enables to have a smooth airgap to avoid high torque ripples. The number of turns of each coil is equal to 73 and the winding is supplied by a 3-phase voltage of $19 \mathrm{~V}$ with a frequency of $1500 \mathrm{~Hz}$. The yoke of the stator is made of FeNi laminations in order to reduce iron losses as the nominal operating point is at $1500 \mathrm{~Hz}$. In fact, this material is better adapted for high speed machines than classical FeSi sheets. The rotor is made up of thin magnetic rings of semi-hard material (Magnetoflex 35) and the stack is surrounding a yoke made of a non-magnetic material. The airgap is very thin compared to the outer diameter of the machine (ratio about $1 / 1000$ ). The picture of the motor is given in Figure 2. 


\section{Presentation of the models}

The numerical model of the motor has to be as simple as possible, so do for the model of material. For the FeNi laminations of the stator, we assume that:

- the eddy currents and hysteresis effects are negligible

- the material is isotropic

The hysteresis loop of the FeNi material is thinner than the characteristic loop of the Magnetoflex. Consequently, the hysteresis effect of FeNi does not influence much the process of the electromechanical conversion.

So, to describe the magnetic behavior of FeNi sheets, we used a single-valued function $H=H(B)$ given by:

$$
H=\frac{B}{\mu_{0}}\left[\frac{B^{2 \alpha}}{B^{2 \alpha}+\tau}(c-\epsilon)+\epsilon\right]
$$

with parameters $(\alpha, \tau, c$ and $\epsilon)$ which can be obtained from experimental measurements. In Figure 3, we give the experimental and calculated anhysteretic curves. The calculated parameters are:

$$
\begin{aligned}
& \alpha=7.3 \\
& \tau=280278000 \text { U.S.I } \\
& \epsilon=1.32 \times 10^{-4} \\
& c=1025
\end{aligned}
$$

However, to model the electromechanical conversion in that kind of machine, it is necessary to take into account the hysteresis effect. In order to choose the adapted hysteresis model, it is necessary to know the material constituting the rotor. The Magnetoflex 35 is little anisotropic and has preferred permanent magnetic properties in the rolling direction. In the transverse direction, the value of

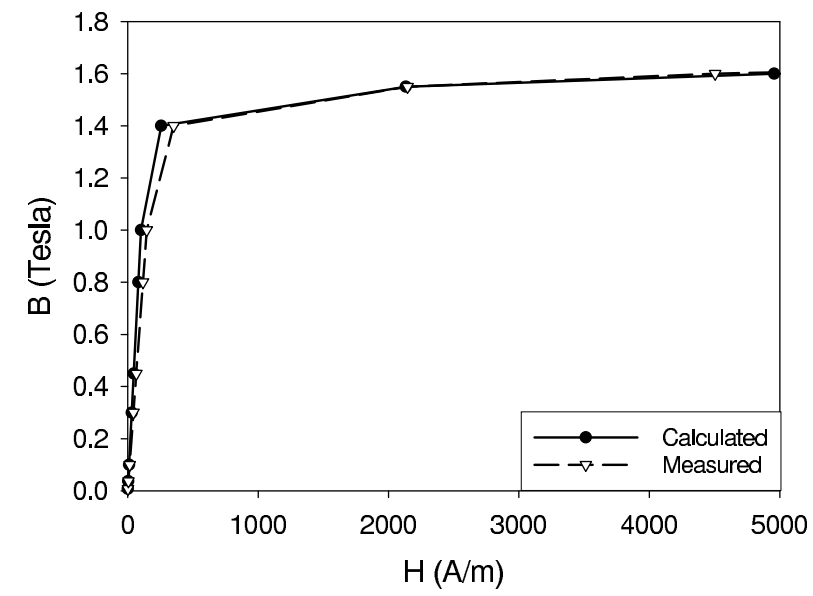

Fig. 3. Anhysteretic curve for the stator

Table 1. Physical properties of Magnetoflex 35

$\begin{array}{ll}\text { Density } & 8.1 \mathrm{~g} / \mathrm{cm}^{3} \\ \text { Electrical resistivity } & 0.7 \times 10^{-6} \Omega . m \\ \text { Curie temperature } & 700{ }^{\circ} \mathrm{C} \\ \text { Remanence } & 0.8-0.95 \mathrm{~T} \\ \text { Coercivity } & 24-30 \mathrm{KA} / \mathrm{m}\end{array}$

the remanent flux density is 10 to $30 \%$ lower. In Table 1, more manufacturer characteristics of the material are given.

To limit the effects of anisotropy, the laminations of the Magnetoflex 35 have been shifted with respect to each other. In this configuration, the lamination stack is assumed to behave like an isotropic medium. It must also 
be noticed that the lamination thickness is small and the hysteresis loop is quite large (the coercive field is 24 to 30 $\mathrm{KA} / \mathrm{m})$. Consequently, the eddy current effect does not modify much the shape of the loop. However, for a motor with a greater thickness of the hysteresis ring, the flux lines are not circumferential and rotational hysteresis occurs in the ring, which increases the iron losses and influences the torque evolution, as already presented in reference [8]. In this case, a vector hysteresis model is needed. In our case, the ring is thin, so the flux lines are mainly circumferential. Then, the directions of $\mathbf{B}$ and $\mathbf{H}$ are only slightly modified and can be considered parallel. Only a relationship linking the modular of $\mathbf{B}$ and $\mathbf{H}$ is needed.

Numerous models for representing the hysteresis phenomenon exist. As it was previously said, the Jiles-Atherton and Preisach based models are the most widely used. From all the above assumptions, we can use an isotropic scalar model to represent the hysteresis effect in the ring. The static Jiles-Atherton model, which is more simple to implement and faster in terms of time calculation [9], has been chosen for this study. In the following, we use a scalar notation for the magnetic field and the magnetic flux density.

The model requires the definition of the anhysteretic curve of the material, which links the magnetic field $H$ to the magnetization $M_{a n}$. It corresponds to the constitutive law of the material when no losses (i.e. no hysteresis) are considered. This curve $M_{a n}(H)$ can be described by a modified Langevin equation:

$$
M_{a n}(H)=M_{\text {sat }}\left[\operatorname{coth}\left(\frac{H_{e}}{a}\right)-\left(\frac{a}{H_{e}}\right)\right]
$$

where $H_{e}=H+\alpha M$ is the effective field experienced by the domains, $H$ is the external applied field, $a$ and $\alpha$ are two parameters. Considering the two contributions in the magnetization process, irreversible $M_{i r r}$ and reversible $M_{\text {rev }}$ magnetization, we write:

$$
\begin{aligned}
& \frac{d M_{i r r}}{d H_{e}}=\frac{\left(M_{a n}-M_{i r r}\right)}{k \delta} \\
& M_{r e v}=c\left(M_{a n}-M_{i r r}\right)
\end{aligned}
$$

where $k$ is a parameter linked to the hysteresis losses and $c$ the reversibility coefficient that belongs to the interval $[0,1]$. The parameter $\delta$ takes the value +1 when $\frac{d H}{d t}>0$ and -1 when $\frac{d H}{d t}<0$. Assuming that the total magnetization is the sum of the reversible and irreversible components, we have the following expression :

$$
M=M_{r e v}+M_{i r r}
$$

with $M_{i r r}$ and $M_{r e v}$ defined by (3) and (4). Using (5) and (4) we can write :

$$
M=M_{i r r}+c\left(M_{a n}-M_{i r r}\right)
$$

Then, with $B_{e}=\mu_{0} H_{e}$ and by differentiating this equation with respect to $B$, we obtain the differential equation of the model:

$$
\frac{d M}{d B}=\frac{(1-c) \frac{d M_{i r r}}{d B_{e}}+c \frac{d M_{a n}}{d B_{e}}}{1+\mu_{0}(1-c)(1-\alpha) \frac{d M_{i r r}}{d B_{e}}+\mu_{0} c(1-\alpha) \frac{d M_{a n}}{d B_{e}}}
$$




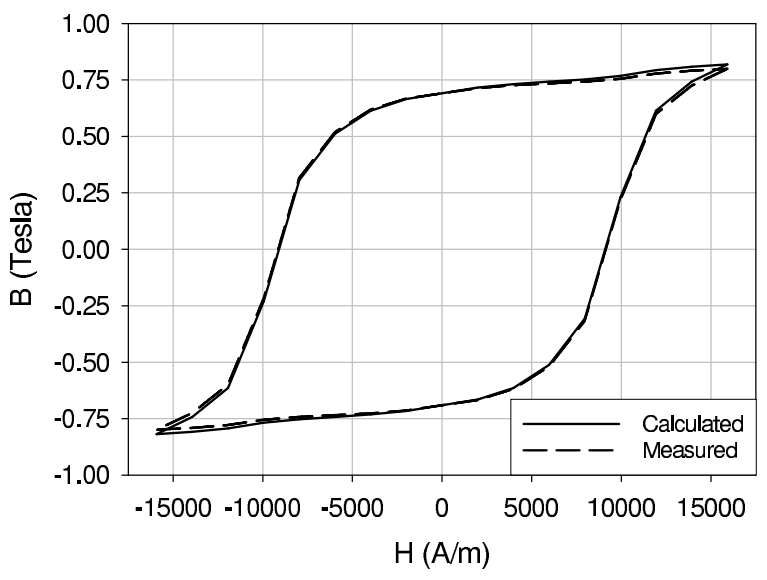

Fig. 4. Magnetoflex 35 hysteresis loop: measurements and model

The five parameters $c, a, k, \alpha$ and $M_{\text {sat }}$ have been identified from only one $B(H)$ loop using an optimization procedure. The obtained values for the Magnetoflex 35 material are the following:

$$
\begin{aligned}
& c=0.25 \text { (reversibility coefficient) } \\
& a=40 \mathrm{~A} / \mathrm{m} \text { (shape factor) } \\
& k=40 \text { (linked to hysteresis losses) } \\
& \alpha=1 \times 10^{-4} \text { (linked to inter-domain coupling) } \\
& M_{\text {sat }}=1200000 \mathrm{~A} / \mathrm{m} \text { (saturation magnetization) }
\end{aligned}
$$

A comparison between the $B(H)$ loops used for the identification and the model is given in Figure 4.

\subsection{Mathematical model}

In the following, the model based on the numerical solution of Maxwell's equation is described. This model takes into account the hysteretic behavior of the ferromagnetic material in the rotor ring and the coupling with external circuit equations. First, we consider the domain $D$ bounding the machine. The surface $S$ corresponds to the surface of the stator. According to all the assumptions, the model is based on magnetostatics equations:

$$
\begin{gathered}
\operatorname{div} \mathbf{B}=0 \\
\text { curl } \mathbf{H}=\sum_{j=1}^{3} \mathbf{J}_{j} \\
\text { with } \mathbf{n . B}=0 \text { on } S_{b} \\
\text { and } \mathbf{n} \wedge \mathbf{H}=\mathbf{0} \text { on } S_{h}
\end{gathered}
$$

with $S_{b}$ and $S_{h}$ two complementary parts of $S, \mathbf{J}_{j}$ the current density flowing the winding $j$ and $\mathbf{n}$ the outward normal vector of $S$. To take into account the material behavior, the constitutive relationship $\mathbf{H}=\mathbf{f}(\mathbf{B})$, is added. To model ferromagnetic material, this relationship can be one of the two models presented previously (equations (1) and (7)). However, these models are scalar models whereas vector models are needed. Then, to obtain this latter, we assume that $\mathbf{B}$ and $\mathbf{H}$ are collinear. The magnitude of $\mathbf{H}$ is calculated from the one of $\mathbf{B}$ and the direction of $\mathbf{H}$ is the same as the one of $\mathbf{B}$. All the previous equations are solved using the $2 \mathrm{D}$ vector potential formulation. As the problem is non-linear, with taking into account hysteresis phenomenon, the fixed point method [10] has been chosen to solve it.

The hysteretic constitutive relationship is then rewritten in the form:

$$
\mathbf{H}=\mathbf{f}(\mathbf{B})=\nu_{F P} \mathbf{B}-\mathbf{M}_{F P}(\mathbf{B})
$$

The reluctivity $\nu_{F P}$ is a constant and must respect some conditions to ensure convergence [11]. The studied 
hysteretic model assumes $\mathbf{B}$ and $\mathbf{H}$ collinear, consequently the magnetization $\mathbf{M}_{F P}$ has the same direction as $\nu_{F P} \mathbf{B}$. Its magnitude is obtained by calculating $M_{F P}=f(B)-$ $\nu_{F P} B$

From (8a), B is derived from a vector potential A such as:

$$
\begin{gathered}
\mathbf{B}=\operatorname{curl} \mathbf{A} \\
\text { withn } \wedge \mathbf{A}=\mathbf{0} \text { on } S_{b}
\end{gathered}
$$

Combining equations (8a) and (8b), the equation to be solved is:

$$
\operatorname{curl} \nu \operatorname{curl} \mathbf{A}=\sum_{j=1}^{3} \mathbf{J}_{j}+\operatorname{curl} \mathbf{M}_{F P}
$$

where $\nu$ is the reluctivity. In the hysteretic medium, the behavior is described by (9), then the reluctivity is equal to $\nu_{F P}$. Elsewhere, $\nu$ corresponds to the actual reluctivity of the model and $M_{F P}$ is equal to zero.

According to the weighting residual method, a weak formulation of (11) can be deduced:

$$
\begin{gathered}
\int_{\mathcal{D}} \nu(\operatorname{curl} \mathbf{A}) \cdot \operatorname{curl} \mathbf{A}^{\prime} d \mathcal{D}= \\
\int_{\mathcal{D}} \mathbf{J} \cdot \mathbf{A}^{\prime} d \mathcal{D}+\int_{\mathcal{D}}\left(\operatorname{curl} \mathbf{M}_{F P}\right) \cdot \mathbf{A}^{\prime} d \mathcal{D}
\end{gathered}
$$

where $\mathbf{A}^{\prime}$ stands for a test function. Boundary conditions will be fixed in a weak sens by vanishing the surface integral in equation (12).

In the $2 \mathrm{D}$ discrete domain, the vector potential is decomposed in the nodal element space. If $\lambda_{i}(x, y)$ is the shape function associated to the node $i$ of the mesh, then:

$$
A(x, y)=\sum_{i=1}^{N} A_{i} \lambda_{i}(x, y)
$$

with $N$ the number of nodes and $A_{i}$ the value of $A(x, y)$ at the node $i$. Applying the Galerkin method to the equation (12). A matrix system is obtained:

$$
[S][A]=[J]+\left[M_{F P}\right]
$$

where the vector $[A]$ represents the nodal values of vector potential $\left(A_{i}\right.$ of $\left.(13)\right),[S]$ a square matrix called stiffness matrix, $\left[M_{F P}\right]$ and $[J]$ the vectors which take into account the magnetization $\mathbf{M}_{F P}$ and the current density J. The non-linearities introduced by hysteretic mediums are reported in the source term $\left[M_{F P}\right]$ which depends on $\mathbf{B}$ (i.e. A).

To take into account the electric circuit, the following equation is added for each winding $\gamma$ of the stator:

$$
U_{\gamma}=R i_{\gamma}+\frac{d \phi_{\gamma}}{d t}
$$

with $U$ the supply voltage, $R$ the resistance of each winding, $i_{\gamma}$ the current and $\phi_{\gamma}$ the flux linkage. To represent the current density, the vector of turns density $\mathbf{N}_{\gamma}$ [12] is introduced for each phase:

$$
\mathbf{J}_{\gamma}=\mathbf{N}_{\gamma} i_{\gamma}
$$

The magnitude of the vector of turns density is given by the ration of the number of turns to the winding section and its direction by the spatial orientation of the winding [12]. On can note that outside the winding $\gamma$ we have $\mathbf{N}_{\gamma}=\mathbf{0}$. The flux linkage in the winding is obtained by 
integrating, in the conductor volume $v$, the projection of the magnetic vector potential on the vector turns density:

$$
\phi_{\gamma}=\int_{v} \mathbf{N}_{\gamma} \cdot \mathbf{A} d v
$$

Then, to take into account the coupling with the external circuit of a coil made up of stranded conductors flowed by a current $i$, a matrix $[D]$ is introduced [12] such that:

$$
[J]=[D][i]
$$

where $[i]$ is the vector of current. Then, combining (14) and (18), the system to be solved becomes:

$$
\left[\begin{array}{cc}
S_{F P} & -D \\
0 & R
\end{array}\right] \cdot\left[\begin{array}{l}
A \\
i
\end{array}\right]+\left[\begin{array}{cc}
0 & 0 \\
D^{t} & 0
\end{array}\right] \cdot \frac{d}{d t}\left[\begin{array}{l}
A \\
i
\end{array}\right]=\left[\begin{array}{l}
0 \\
u
\end{array}\right]+\left[\begin{array}{c}
M_{F P} \\
0
\end{array}\right]
$$

This system can be time discretised using an Euler implicit scheme.

\section{Validation of the model: comparison with measurements}

In the following, we present the results obtained for a mesh composed of 868 elements, which has been found to be sufficiently accurate. An example of the flux pattern obtained in the machine is given in Figure 5.

To validate the model, we propose to compare the measurement with the calculated torque and electrical quantities. The calculations were carried out when the motor is supplied by the nominal voltage (3 phased sinusoidal voltage of $19 \mathrm{~V}$ RMS). First, we give the characteristic for

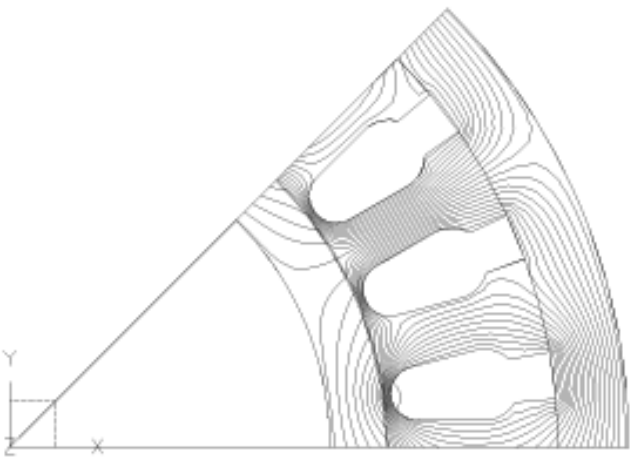

Fig. 5. Flux pattern in the machine

the torque versus the speed measured during a startup of the machine from standstill to the synchronous speed (Figure 6). One can note a variation of the experimental torque of about $20 \%$. The model leads to a constant torque equal to $0.72 m N . m$. In fact,in the simulation, no dynamic effects are considered since eddy currents and frequency dependency of the hysteresis loop are neglected. Under these conditions, the rotor speed has no influence since the shape of the hysteresis loop depends only on the field magnitude, not on its time variation. We can see that the mean value of the computed torque agrees rather well with the experimental one, taking into account that this quantity is generally difficult to calculate for a machine with thin airgap (asynchronous machine, reluctance variable machine,...).

The measured waveforms of the line current and voltage for synchronous speed are given in Figure 7. The waveforms of the calculated current is given in Figure 8. It does not depend on the rotor speed either. For more convenience, we give in Table 2 the magnitude of the fundamental and harmonics of currents for both measurements and calculations. We have also added the values for the exper- 
Please give a shorter version with: \authorrunning and \titlerunning prior to \maketitle

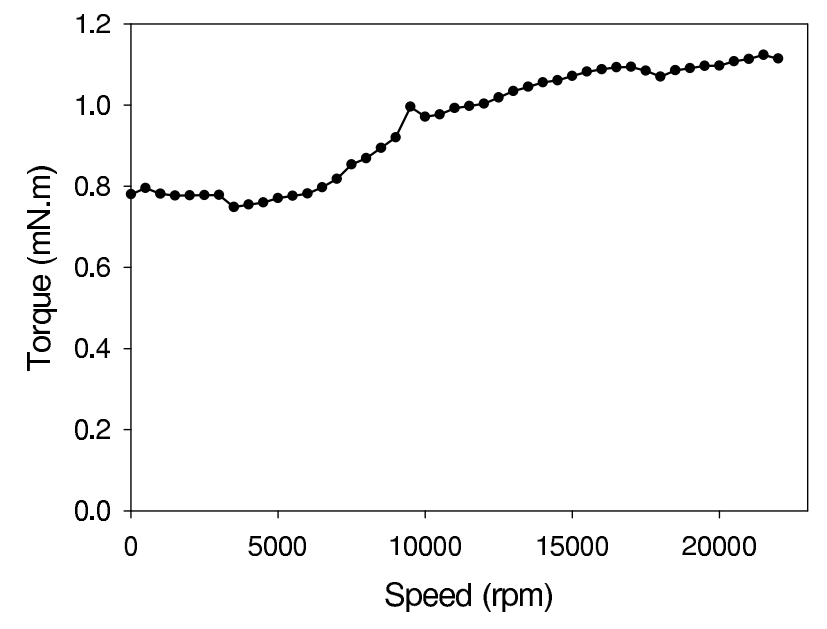

Fig. 6. Experimental evolution of the torque versus the speed during a startup

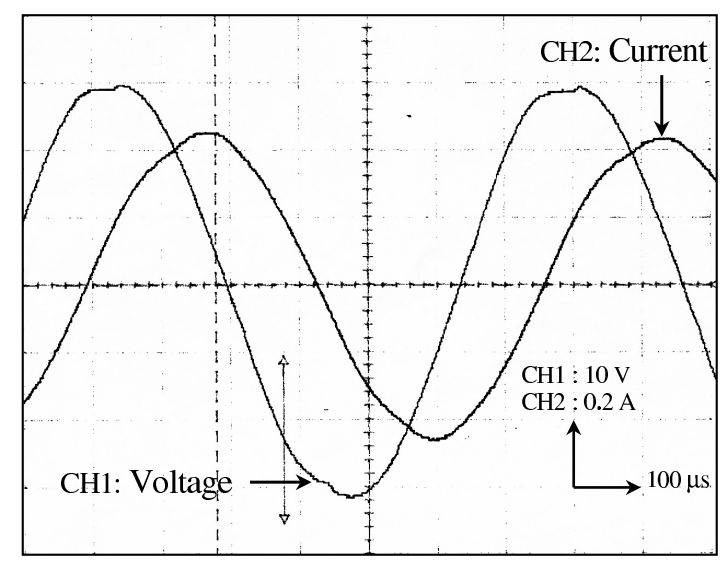

Fig. 7. Measured current and voltage waveshapes at rated speed

imental currents when the motor is supplied at standstill. We can note a difference between both measured currents, at standstill and at synchronous speed, due to dynamic effects which appear mainly at standstill in the rotor.

Results of Table 2 also show that calculation results are quite close to the experimental results. These comparisons show that the assumptions made in the model seem to be justified for this motor. Then, the model can be used to study the influence of parameters on the torque.

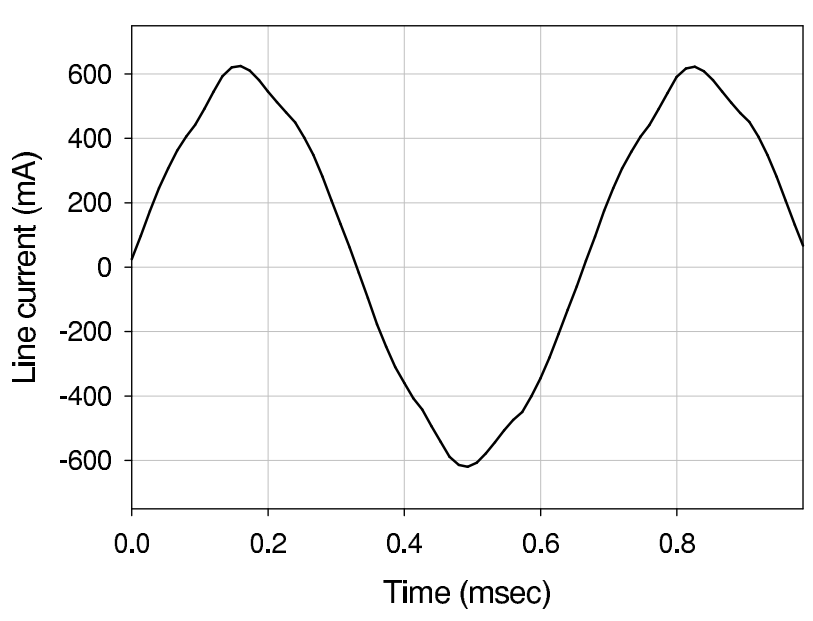

Fig. 8. Time evolution of the line current in one phase

Table 2. Current RMS values (in Ampere) obtained by measurement and field calculation

\begin{tabular}{llll}
\hline & Measurement & Measurement & Calculation \\
& (synchronous speed) & (at standstill) & \\
$I_{R M S}$ & $322.2 \times 10^{-3}$ & $509.6 \times 10^{-3}$ & $417.5 \times 10^{-3}$ \\
$I_{h 1}$ & $322.0 \times 10^{-3}$ & $478.0 \times 10^{-3}$ & $416.9 \times 10^{-3}$ \\
$I_{h 5}$ & $4.33 \times 10^{-3}$ & $18.94 \times 10^{-3}$ & $11.27 \times 10^{-3}$ \\
$I_{h 7}$ & $1.96 \times 10^{-3}$ & $7.73 \times 10^{-3}$ & $7.18 \times 10^{-3}$ \\
\hline
\end{tabular}

In the following section, we present a comparison of the hysteresis motor features when supplying either by imposing a sinusoidal current or by imposing a sinusoidal voltage. 


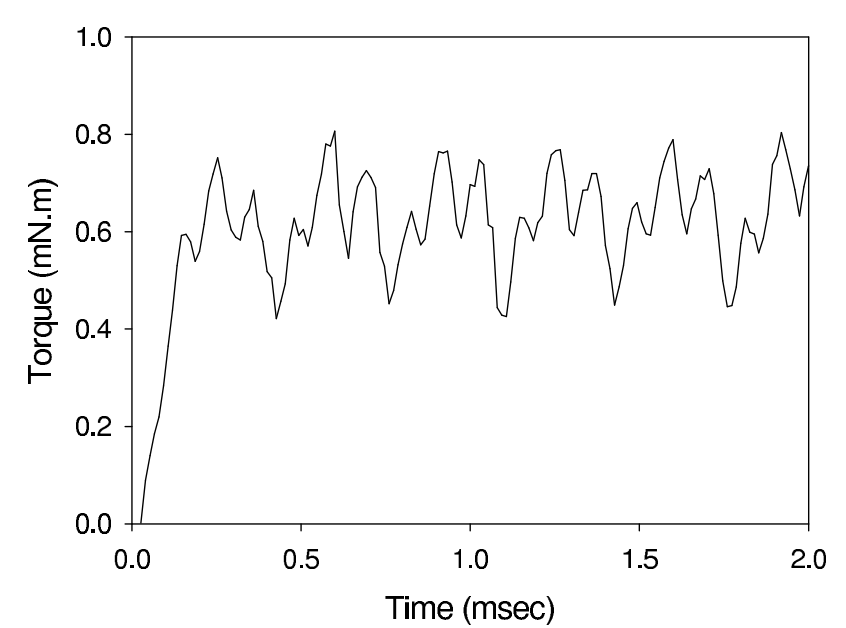

Fig. 9. Time evolution of the torque when imposing a sinusoidal current $I_{\text {line }}=0.44 \mathrm{~A}$

\section{Study of the motor by field calculation}

\subsection{Study of the calculated torque}

In Figures 9 and 10, we give the results obtained for the torque by field calculation by imposing respectively the RMS values for the currents $I_{\text {line }}=0.44 A$ and the voltages $V=19 \mathrm{~V}$ which correspond to the nominal values. Both supplies lead to close average torque values at steady state (Table 3). In both cases, the semi-hard material is initially supposed to be demagnetized ( $M=0$ for $H=0)$.

When supplying by sinusoidal current (Fig. 9), in the first part of the curve we can see the magnetization process and in the second part the steady state when the magnitude of the torque ripple is about $50 \%$ of the average torque. In Figure 10, when the motor is supplied by sinusoidal voltages, we observe a longer transient. But, in this case, the torque ripple magnitude is weaker and represents about $25 \%$ of the average torque.

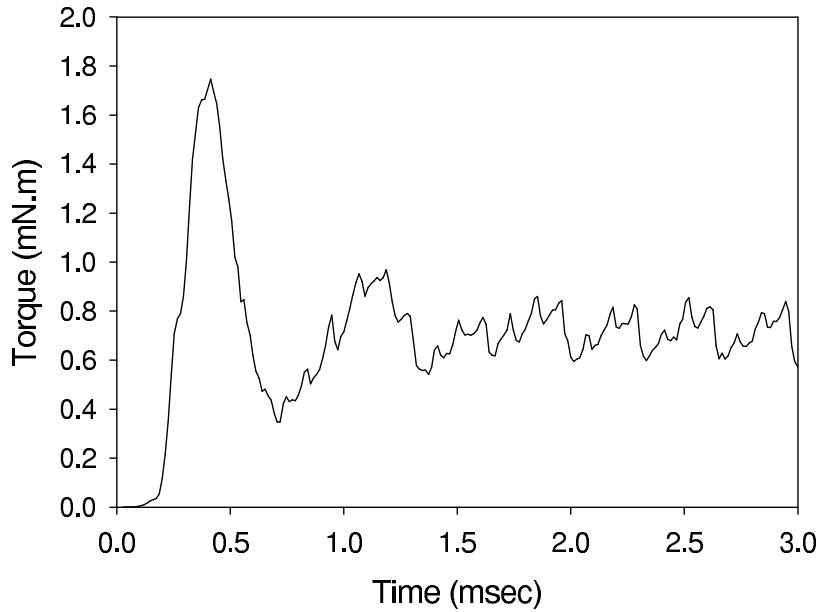

Fig. 10. Time evolution of the torque when imposing a sinusoidal voltage $V=19 \mathrm{~V}$

Table 3. Torque values obtained by field calculation

\begin{tabular}{|c|c|}
\hline Calculation (imposed current) & $0.65 \mathrm{mN} . \mathrm{m}$ \\
\hline Calculation (imposed voltage) & $0.72 m N . r$ \\
\hline
\end{tabular}

We have also computed the evolution of the average torque versus the current and the voltage magnitudes (Figures 11 and 12) at steady state. We observe a quadratic evolution of the torque versus the applied current, i.e. the applied magnetic field. Nevertheless, if the current is too high, due to the magnetic saturation, the torque rate evolution versus the current becomes slower. This case cannot appear in practice as it leads to an overheating of the machine due to an excess of Joule losses. 


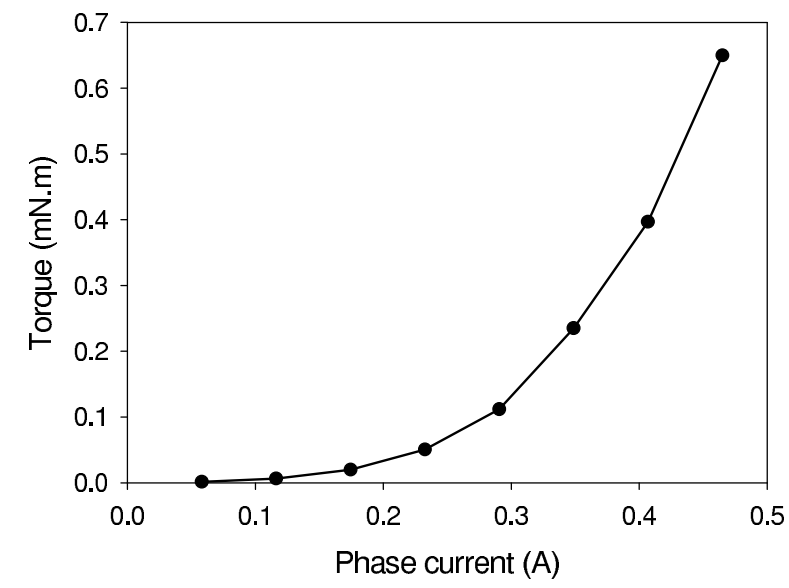

Fig. 11. Evolution of the torque versus the supply current

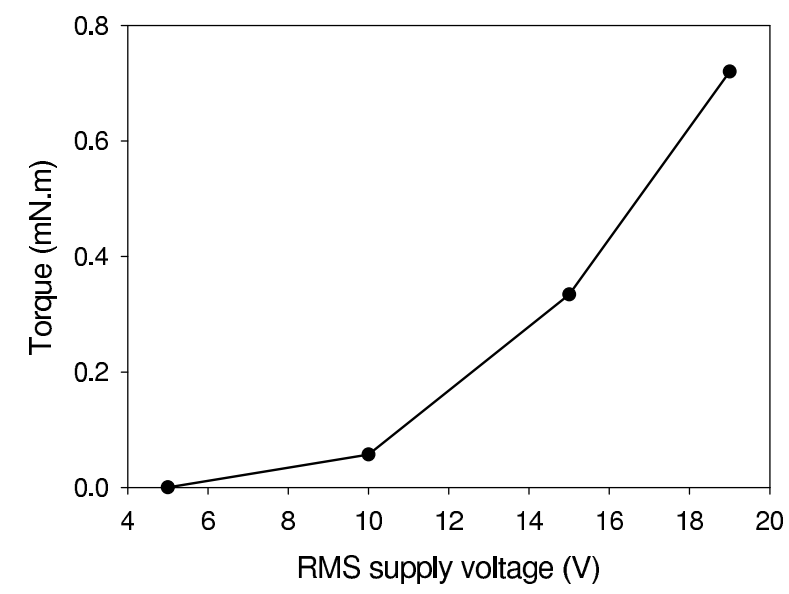

Fig. 12. Evolution of the torque versus the supply voltage

The evolution of the torque versus the applied voltage is given in Figure 12. We notice a similar evolution.

\subsection{Current waveforms when applying the voltages}

The phases are coupled in delta, so the line currents are a combination of phase currents. When the supply voltage is sinusoidal, the line currents and the phase currents are not sinusoidal as seen above in section 4 . The harmonic distorsion of line currents remains small compared to the one of the current flowing the windings (Figure 13) which

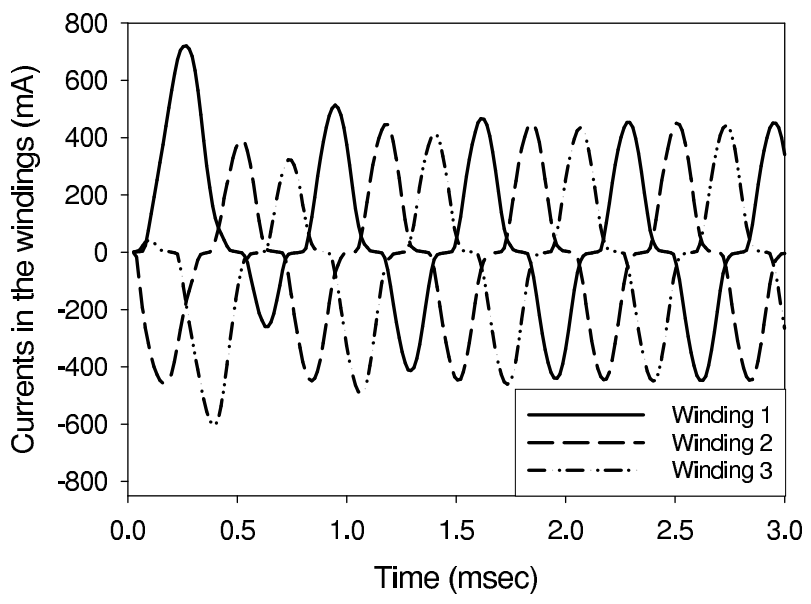

Fig. 13. Currents in the windings when imposing the voltage

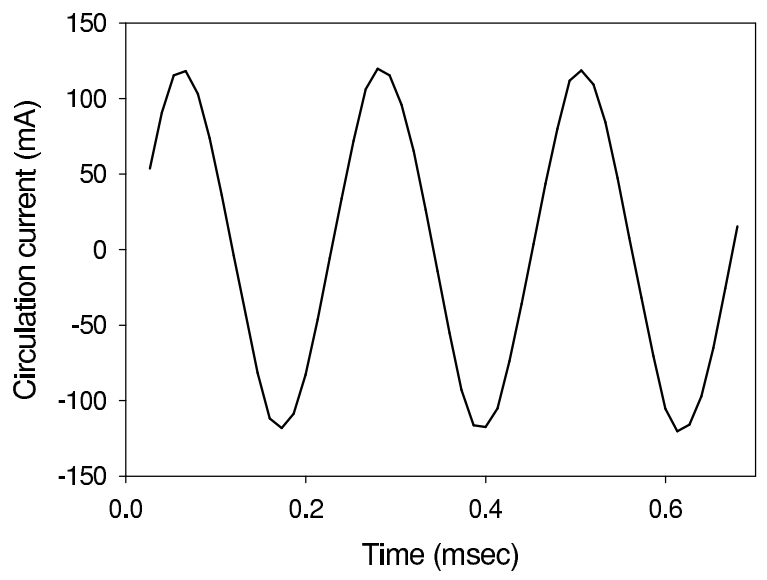

Fig. 14. Circulation current in the delta coupled winding for one fundamental period

third harmonic has a magnitude of $115 \mathrm{~mA}$. Due to the saturation and hysteresis effects, an important circulation current appears, as shown in Figure 14. This circulation current lead to a reduction of the torque ripple. Indeed, we have noticed that the ripple magnitude are greater when the current is imposed with a perfect sinusoidal waveform. 


\section{Conclusion}

We have shown in this paper that modelling of the presented hysteresis motor can be achieved in finite element analysis with a quite good accuracy when considering only a static scalar hysteresis model. The presented model has been validated by comparison with measurements in terms of torque and electrical quantities. The model has been also used to predict the hysteresis motor behavior when it is supplied with a sinusoidal current or voltage of variable magnitudes. A quadratic evolution of the torque has been found. This model is sufficiently accurate and fast to be used in the design of a hysteresis motor.

\section{References}

1. F. Preisach, Über die magnetische nachwirfung. Zeitschrift für Physik vol. 94, (1935) pp. 277-302.

2. I.D. Mayergoyz, Mathematical models of hysteresis (Springer Verlag - New York, 1991).

3. D.C. Jiles and D.L. Atherton, Journal of Magnetism and Magnetic Materials vol. 61, (1986) pp. 48-60.

4. L. Dupré, J. Gyselinck and J. Melkebeek, IEEE Trans. on Magnetics vol. 34, No. 5, (1998) pp. 3048-3051.

5. A. Rahman, Analytical models for polyphase hysteresis motor, IEEE Winter Power, December 10 (1970).

6. G.R. Siemon, R.D. Jackson and M.A. Rahman, Trans. on Power Apparatus and Systems vol. PAS-96, No. 6, (1977).

7. H-K. Kim, S-K. Hong and H-K. Jung, IEEE Trans. on Magnetics vol. 36, No. 4, (2000) pp. 685-688.

8. S-K. Hong, H-K. Kim, H-S. Kim and H-K. Jung, IEEE

Trans. on Magnetics vol. 36, No. 4, (2000) pp. 685-688.
9. A. Benabou, S. Clénet and F. Piriou, Journal of Magnetism and Magnetic Materials vol. 261, Issues 1-2, (2003) pp. 139-160.

10. O. Bottauscio, D. Chiarabaglio, M. Chiampi and M. Repetto, IEEE Trans. on Magnetics vol. 31 No. 6, (1995) pp. $3548-3550$.

11. V. Ionita, B. Cranganu-Cretu and B. Iona, IEEE Trans. on Magnetics vol. 32, No. 3, (1996) pp. 1128-1131.

12. F. Piriou, A. Razek, IEEE Trans. on Magnetics vol. 29, (1993) pp. 1669-1675. 